\title{
Salidroside inhibits steroid-induced avascular necrosis of the femoral head via the PI3K/Akt signaling pathway: In vitro and in vivo studies
}

\author{
XING-HE XUE, ZHEN-HUA FENG, ZHEN-XING LI and XIAO-YUN PAN
}

\begin{abstract}
Department of Orthopaedics, The Second Affiliated Hospital and Yuying Children's Hospital of Wenzhou Medical University, Wenzhou, Zhejiang 325000, P.R. China
\end{abstract}

Received August 22, 2017; Accepted December 14, 2017

DOI: $10.3892 / \mathrm{mmr} .2017 .8349$

\begin{abstract}
Dexamethasone (Dex) and other glucocorticoids are widely used to treat serious infections and immunological diseases, however they may cause steroid-induced avascular necrosis of the femoral head (SANFH). Salidroside (Sal) has demonstrated an anti-apoptotic effect on neurocytes by activating the phosphoinositide 3 -kinase (PI3K)/protein kinase B (Akt) signaling pathway. In the present study, primary osteoblasts were used in vitro and in rats in vivo to determine the anti-apoptotic effect of Sal on SANFH. The result of the present study demonstrated that pretreatment with Sal increased the cell survival rate while decreasing the cell apoptosis and lactate dehydrogenase release rate. Additionally, Sal also caused the reduction of TUNEL positive cells in TUNEL staining assay. Sal decreased the expression of cleaved caspase-3, cleaved caspase-9, apoptosis regulator BAX and cytochrome $\mathrm{C}$, while it increased the expression of B cell lymphoma-2 and phosphorylated-Akt in Dex-induced osteoblasts. In vivo Sal protected against SANFH in rats by decreasing the percentage of empty lacunae. The present study demonstrated that Sal alleviated Dex-induced osteoblast apoptosis by activating the PI3K/Akt signaling pathway and downregulating caspase-3 expression in osteoblasts. Sal also protected against SANFH in a rat model of SANFH by decreasing the percentage of empty lacunae. The inhibition of the mitochondrial apoptosis pathway was also involved. Further research is required to determine the full underlying mechanisms by which Sal has an effect.
\end{abstract}

Correspondence to: Dr Xiao-Yun Pan, Department of Orthopaedics, The Second Affiliated Hospital and Yuying Children's Hospital of Wenzhou Medical University, 109 Xueyuan Xi Road, Wenzhou, Zhejiang 325000, P.R. China

E-mail: starriver789@163.com

Key words: steroid-induced avascular necrosis of the femoral head, osteoblast, apoptosis, salidroside, phosphoinositide 3-kinase/protein kinase B signaling pathway

\section{Introduction}

Steroid-induced avascular necrosis of the femoral head (SANFH), as the most common type of femoral head necrosis, also leads to femoral head collapse and osteoarthritis if not being treated properly $(1,2)$. Finally, most patients require total hip arthroplasty. Nowadays, Dexamethasone (Dex) and other glucocorticoids (GCs) are of great importance in curing many diseases such as serious infection, inflammation, anti-shock, allergic disease, hematological system diseases and replacement therapy (3-6). Since the wide application of Dex and other GCs, the incidence of SANFH has already exceeded trauma-induced femoral head necrosis (7). However, the pathogenesis of SANFH is still expecting for further research. It is urgent to clarify the pathogenesis of SANFH, and find out a proper treatment for SANFH.

The imbalance between osteoblast and osteoclast activity, by inhibiting osteoblastogenesis and promoting osteoblast apoptosis, will finally cause osteonecrosis $(8,9)$. Osteoblast, which is the main target of Dex and other GCs, plays a vital role in promoting bone formation $(10,11)$. Dex-induced osteoblasts apoptosis was used to create a cellular model of SANFH, which has been proved to be feasible in our recent study (12).

Salidroside (Sal), which is one important medicinal ingredient of Rhodiola rosea $\mathrm{L}$, is useful in anti-aging, memory improvement, anti-fatigue, anti-oxidation, anti-depressant and so on (13-17). Previous studies found Sal could reduce apoptosis induced by glutamate in rat primary cultured hippocampal neurons (18). Sal attenuates colistin-induced neurotoxicity in RSC96 Schwann cells via activating the phosphoinositide 3-kinase $(\mathrm{PI} 3 \mathrm{~K}) /$ protein kinase $\mathrm{B}(\mathrm{Akt})$ signaling pathway and downregulating caspase-3 expression (19). The PI3K/Akt pathway is a critical pathway related to several fundamental cellular processes, such as survival, proliferation, growth and differentiation (20-24). Recently, a study showed that the suppression of the PI3K/Akt signaling pathway caused osteoblast damage (25). Caspase-3, a subtype of the caspase proteins as dormant proenzymes in cells, cleaves cellular substrates. Caspase- 3 also causes cell apoptosis whether it is activated by the intrinsic pathway or the extrinsic pathway $(26,27)$.

In our study, we employed primary osteoblasts in vitro and rats in vivo to find out the anti-apoptosis effect of Sal 
on SANFH. Furthermore, we also tried to explore the mechanism of the protective effect of Sal. We assumed Sal had an anti-apoptosis effect on SANFH via activating the PI3K/Akt signaling pathway and subsequently downregulating the caspase- 3 expression.

\section{Materials and methods}

Preparation of primary murine osteoblasts. According to our previous study (12), osteoblasts were isolated and extracted by digesting neonatal rats' calvariae using collagenase I (Sigma-Aldrich, St. Louis, MA, USA). Digests 3-6 were neutralized, pooled, and filtered. After we resuspended the single-cell suspensions, the single-cell suspensions was cultured in alpha-Minimal Essential Medium ( $\alpha$-MEM) which supplemented with $12 \%$ fetal bovine serum (FBS) and antibiotics. The operation procedures were approved by the Animal Care and Use Committee at our institution and complied with the guidelines of the international regulations.

Cell survival assay, cell apoptosis assay and $L D H$ release assay. The Cell Counting kit-8 (CCK-8; Dojindo Laboratories, Kumamoto, Japan) and Cell Apoptosis-DNA ELISA Plus kit (Roche, Palo Alto, CA, USA) was respectively used as quantitative measurement to assess the cell survival and the cell apoptosis. We recorded both the optic density (OD) of each well at $450 \mathrm{~nm}$.

The lactate dehydrogenase (LDH) in the conditional medium was also measured by a two-step enzymatic reaction LDH assay kit (Takara, Tokyo, Japan) to assess the cell apoptosis. The percentage of $\mathrm{LDH}$ release $=\mathrm{LDH}$ (conditional medium) $/ \mathrm{LDH}$ (conditional medium + cell lysates).

TUNEL assay. We used TUNNEL staining to detect the apoptotic osteoblasts by the In Situ Cell Death Detection kit (Roche). The cultured osteoblasts were fixed with freshly $4 \%$ paraformaldehyde for $1 \mathrm{~h}$ in a six-well plate. Then they were incubated with $3 \% \mathrm{H}_{2} \mathrm{O}_{2}$ and $0.1 \%$ Triton $\mathrm{X}-100$ for $10 \mathrm{~min}$. During each step, they were washed three times with phosphate buffered saline (PBS). Finally, cells were stained with DAPI. A fluorescence microscope (Olympus, Tokyo, Japan) was used to obtain the images and assess the apoptotic cells. The TUNEL positive cells were calculated by three different authors.

Western blot analysis. As described (12), we used six-well plates to culture osteoblasts $\left(3 \times 10^{5}\right.$ cells/well) and added Dex or/and Sal at different time. In the Sal group and Dex + Sal group, osteoblasts were pretreated with the optimum concentration of Sal (100 $\mathrm{nM})$ for $2 \mathrm{~h}$ before the addition of Dex. We used radioimmunoprecipitation assay (RIPA) buffer containing phenylmethane sulfonyl fluoride (PMSF) to extract proteins from the osteoblasts. Then the protein concentration was calculated by the BCA Protein Assay kit (Beyotime Biotechnology, China). $12 \%$ sodium dodecyl sulfate polyacrylamide gel electrophoresis (SDS-PAGE) was adopted to resolve each protein lysate samples (30 $\mu \mathrm{g}$ in average). Then the solution was transferred onto polyvinylidene fluoride (PVDF) membranes. After the membranes were blocked and incubated with the primary antibody $(1: 1,000)$ overnight, they were incubated with each secondary antibody
$(1: 3,000)$ for $2 \mathrm{~h}$. Electrochemiluminescence Plus Reagent (Invitrogen Life Technologies, Carlsbad, CA, USA) was used to make the bands visible. We also used Image Lab 3.0 software (Bio-Rad, Hercules, CA, USA) to assess the band intensity. All the primary antibodies against cleaved-caspase-3, cleaved caspase-9, BAX, Bcl-2, cytochrome C, Akt and phosphor-Akt (Ser473) were purchased from Cell Signaling Technology (Danvers, MA, USA).

Rat model in vivo. We chose 12-weeks-old adult male Sprague-Dawley rats (Animal Center of the Chinese Academy of Sciences, Shanghai, China) to build our animal model. We divided 45 rats equally into three groups (the control group, the model group and the Sal group). According to our previous study (12), The experimental rats in the model group and the Sal group were administered with double doses of lipopolysaccharide (LPS; Escherichia coli oup:B5; Sigma-Aldrich) on day 0 and 1 and received intramuscular injection of $40 \mathrm{mg} / \mathrm{kg}$ methlprednisolone (MPS) sodium succinate (Pfizer Pharmaceutical, Beijing, China) on day 3,4, and 5. The rats in the control group were injected with normal saline (NS) instead. Then, the rats in the Sal group received an intraperitoneal injection of Sal $(25 \mathrm{mg} / \mathrm{kg} / \mathrm{d}$ ) for seven consecutive days. The rest of the rats were injected with NS. All the injections were carried out at the same time each day and the time interval was $24 \mathrm{~h}$.

We used 50×35× $20 \mathrm{~cm}$ plexiglass cages to raise the rats. We also controlled the laboratory conditions (relative humidity of $50 \pm 10 \%$, constant temperature of $20 \pm 2^{\circ} \mathrm{C}$ and $12 \mathrm{~h} \mathrm{light/dark}$ cycle). All the rats were allowed free access to food and water. 8 weeks after the final injection of MPS, all the rats were sacrificed and bilateral femoral heads were collected for histological analysis. Our protocol was carried out in accordance with the National Institutes of Health guide for the care and use of Laboratory animals and was also approved by the Animal Care and Use Committee of Wenzhou Medical University.

Histological analysis. Femoral head samples, collected from rat models in vivo, were fixed in paraformaldehyde for $24 \mathrm{~h}$ at $4^{\circ} \mathrm{C}$. Then we transferred the samples to $10 \%$ EDTA solution at $4^{\circ} \mathrm{C}$ for 2 months. After the samples were dehydrated in ethanol, the samples were embedded in paraffin and cut into $4-\mu \mathrm{m}$ thick sections in the coronal plane. We used hematoxylin-eosin for tissue section staining. With a microscope (Leica Microsystems, Wetzlar, Germany), we observed the pyknotic nuclei of osteocytes, empty lacunae, bone marrow cell necrosis and bone trabeculae broken. The percentage of empty lacunae was assessed blindly by three authors.

Statistical analysis. We repeated all the experiments at least three times with consistent outcomes. We used SPSS 21.0 software (IBM SPSS, Armonk, NY, USA) to convert all outcome measurements. The one-way ANOVA followed by Tukey's post hoc test was used to calculate statistical significance, and $\mathrm{P}<0.05$ was considered to indicate a statistically significant difference.

\section{Results}

In our previous study (12), we found Dex induced osteoblast apoptosis related to dose and time. Cells exposed to $1 \mu \mathrm{M}$ Dex 
A
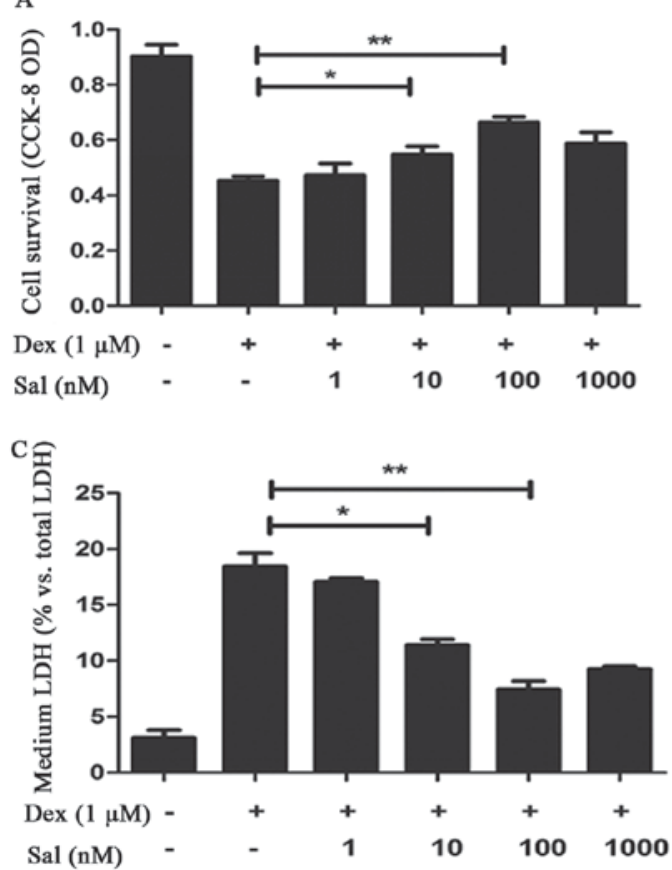

B

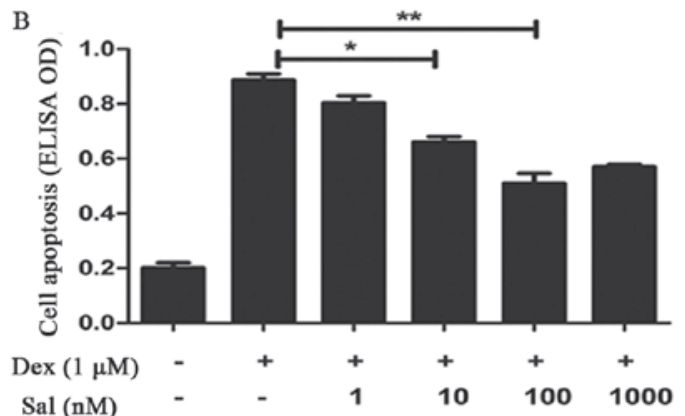

$\mathrm{D}$

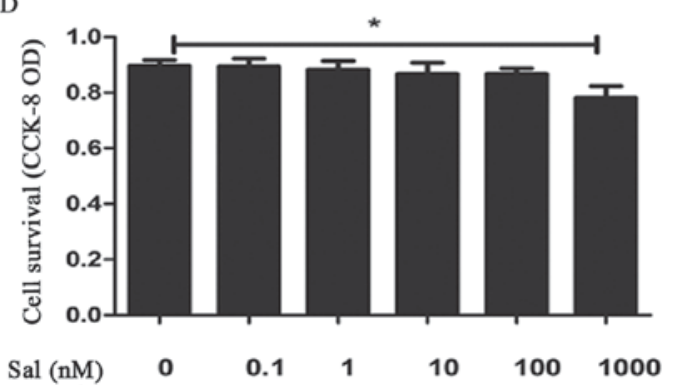

Figure 1. Sal protects osteoblasts from Dex-induced damage. Primary murine osteoblasts were pretreated with the indicated concentrations of Sal $(0-1,000 \mathrm{nM})$ for $2 \mathrm{~h}$, followed by treatment with Dex $(1 \mu \mathrm{M})$. Cells were then cultured in conditional medium for $24 \mathrm{~h}$, after which cell survival and apoptosis were measured by the CCK-8 assay (A and D) and histoneDNA ELISA (B). Furthermore, the LDH release was assayed (C). Sal showed a significant protective effect on cell survival assay and against Dex-induced cell death (cell apoptosis assay and LDH release assay) when the concentration reached $10 \mathrm{nM}(\mathrm{P}<0.05)$ and $100 \mathrm{nM}(\mathrm{P}<0.01)(\mathrm{A}-\mathrm{C})$. Respectively, Sal had no potential cytotoxic effect when the concentration was not more than $100 \mathrm{nM}$ $(\mathrm{P}<0.05)(\mathrm{D})$. Data are presented as the mean \pm standard deviation. Experiments in this figure were repeated five times. Significant differences between two groups are indicated as ${ }^{* *} \mathrm{P}<0.01$ and ${ }^{*} \mathrm{P}<0.05$. Sal, salidroside; Dex, dexamethasone; CCK- 8 , cell counting kit- 8 ; OD, optical density; LDH, lactate dehydrogenase.

for 24 h obviously damaged cell viability, and the dose and time of Dex were used in our subsequent experiments.

Sal attenuates Dex-induced osteoblasts apoptosis. Sal showed a significant protective effect on cell survival assay and against Dex-induced cell death (cell apoptosis assay and $\mathrm{LDH}$ release assay) when the concentration reached $10 \mathrm{nM}$ $(\mathrm{P}<0.05)$. And the protective effect of Sal was most obvious at $100 \mathrm{nM}(\mathrm{P}<0.01)$ (Fig. 1A-C). Furthermore, we used CCK-8 to value the cytotoxic effect of Sal. As shown in Fig. 1D, Sal had no potential cytotoxic effect on the primary murine osteoblasts in Sal alone group, which reflected in the decline of CCK-8 OD, when the concentration was not more than $100 \mathrm{nM}(\mathrm{P}<0.05)$.

The TUNEL assay revealed that TUNEL positive cells significantly increased when treated with Dex $(\mathrm{P}<0.01)$ (Fig. 2). And the pretreatment of Sal obviously decreased TUNEL positive cells compared with the Dex group $(\mathrm{P}<0.01)$ (Fig. 2). What's more, there is no significant difference between the Sal alone group and the control group $(\mathrm{P}>0.05)$.

Sal alleviated Dex-induced osteoblasts apoptosis via activating the PI3K/Akt signaling pathway and downregulating the caspase protein expression. To explore the mechanism by which Sal decreased Dex-induced apoptosis, western blot analysis was performed to study changes in the PI3K/Akt signaling pathway. As shown in Fig. 3A, B, Dex significantly decreased p-Akt level in the Dex alone group compared with the control group $(\mathrm{P}<0.01)$. Pretreatment with $\mathrm{Sal}$ for
$2 \mathrm{~h}$ significantly increased p-Akt expression in Dex-induced osteoblasts apoptosis $(\mathrm{P}<0.01)$. And the expression of $\mathrm{p}$-Akt in the Sal alone group did not show any change compared with the control group $(\mathrm{P}>0.05)$. Total Akt levels in each group did not change.

To further investigate the effect of PI3K/Akt signaling in the caspase protein expression, cells were pretreated with Sal for $2 \mathrm{~h}$ and then stimulated with Dex for $24 \mathrm{~h}$. Sal significantly downregulated the expression of Dex-induced cleaved caspase-9 and cleaved caspase-3 ( $\mathrm{P}<0.01)$ (Fig. 3C, D).

Sal protected against Dex-induced osteoblasts apoptosis by inhibiting the mitochondrial apoptosis pathway. We used western blot analysis to explore whether the mitochondrial pathway is involved in the Sal-induced protective effect against Dex-induced osteoblasts apoptosis. The expression levels of BAX, Bcl-2 and cytochrome $\mathrm{C}$ were detected. As shown in Fig. 4, compared with the control group, the expression level of pro-apoptotic protein Bax significantly increased $(\mathrm{P}<0.01)$ while the expression level of anti-apoptotic protein Bcl-2 was on the contrary in the Dex group after exposure to Dex for $24 \mathrm{~h}(\mathrm{P}<0.01)$. Pretreatment with Sal for $2 \mathrm{~h}$ significantly decreased BAX expression and increased $\mathrm{Bcl}-2$ expression in Dex-induced osteoblasts apoptosis $(\mathrm{P}<0.01)$. Moreover, we found cytochrome $\mathrm{C}$ increased significantly by Dex $(\mathrm{P}<0.01)$, but the expression could be inhibited by Sal $(\mathrm{P}<0.01)$. And the expression of BAX, Bcl-2 and cytochrome $\mathrm{C}$ in Sal alone group did not show any change compared with the control group. 
A

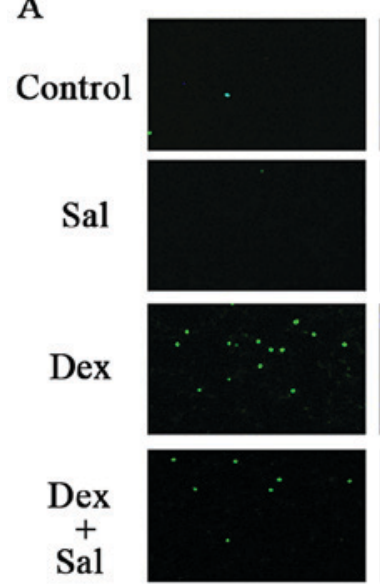

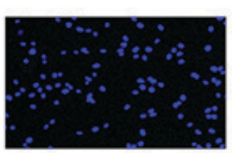
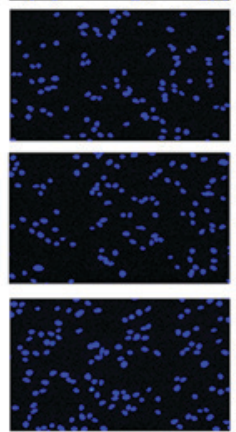

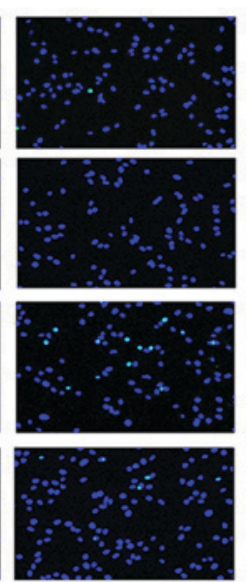

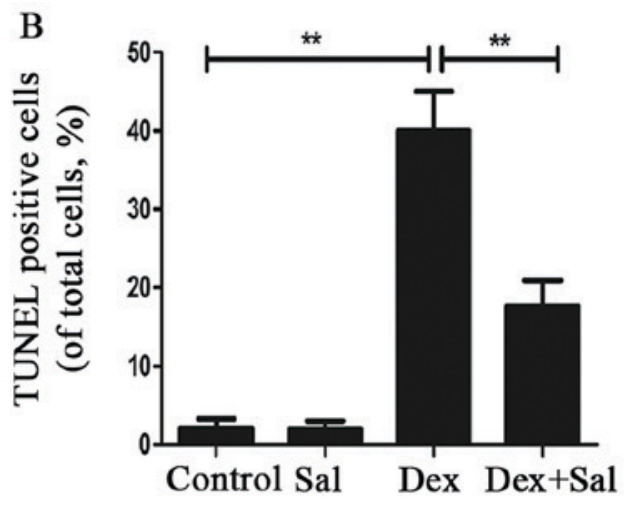

Figure 2. Sal attenuates Dex-induced osteoblasts apoptosis by TUNEL assay. The TUNEL assay revealed that TUNEL positive cells significantly increased when treated with Dex $(\mathrm{P}<0.01)$, and the pretreatment of Sal obviously decreased TUNEL positive cells compared with the Dex group $(\mathrm{P}<0.01)(\mathrm{A}$ and $\mathrm{B})$. Significant differences between two groups are indicated as ${ }^{* *} \mathrm{P}<0.01$. Sal, salidroside; Dex, dexamethasone.
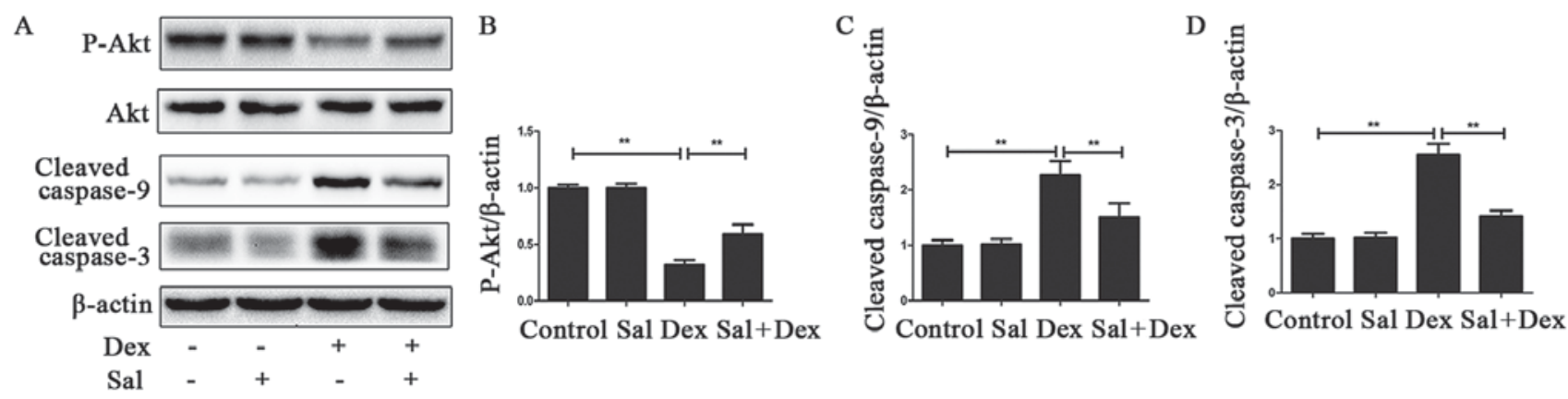

Figure 3. Sal alleviates Dex-induced osteoblasts apoptosis via activating PI3K/Akt-mediated downregulation of caspase-3 expression. (A) The protein expression of Akt, p-Akt, cleaved caspase-9 and cleaved caspase-3 in osteoblasts was determined by western blot analysis. The results of the western blot were quantified and the protein expression of (B) p-Akt, (C) cleaved caspase-3 and (D) cleaved caspase-9 were determined. Data represent the average \pm SD. Significant differences between two are indicated as ${ }^{* *} \mathrm{P}<0.01$. Sal, salidroside; Dex, dexamethasone; Akt, protein kinase B; p, phosphorylated; PI3K, phosphoinositide 3-kinase.
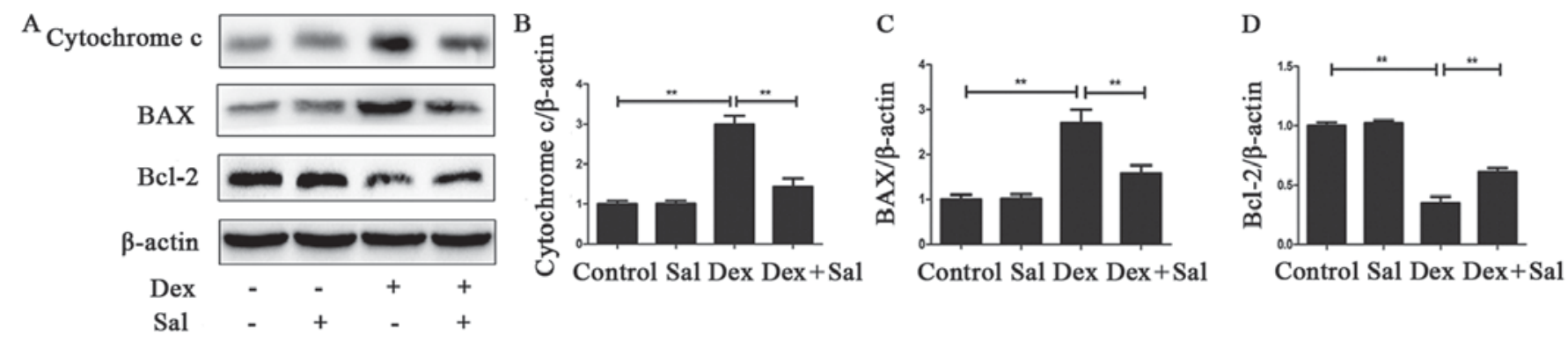

Figure 4. The mitochondrial pathway is involved in Dex-induced osteoblasts apoptosis. (A) The protein expression of BAX, Bcl-2 and cytochrome C in osteoblasts was determined by western blot analysis. The results of the western blot were quantified and the protein expression of (B) cytochrome C, (C) BAX and (D) Bcl-2 were determined. Data represent the average \pm SD. Significant differences between two groups are indicated as ${ }^{* * *} \mathrm{P}<0.01$. Sal, salidroside; Bcl-2, B cell lymphoma 2; BAX, apoptosis regulator BAX; Dex, dexamethasone.

Sal protected against SANFH in rats by decreasing the percentage of empty lacunae. The Sal group (2/15) significantly decreased the incidence rate of SANFH compared with the model group $(13 / 15)(\mathrm{P}<0.01)$. As shown in Fig. 5, the percentage of empty lacunae increased significantly in the model group $(\mathrm{P}<0.01)$. Additionally, Sal reduced the percentage of empty lacunae compared with the model group
$(\mathrm{P}<0.01)$ while Sal would not increase the percentage of empty lacunae compared with the control group.

\section{Discussion}

The present study showed that pretreatment with Sal attenuated Dex-induced osteoblasts apoptosis by increasing cell survival 

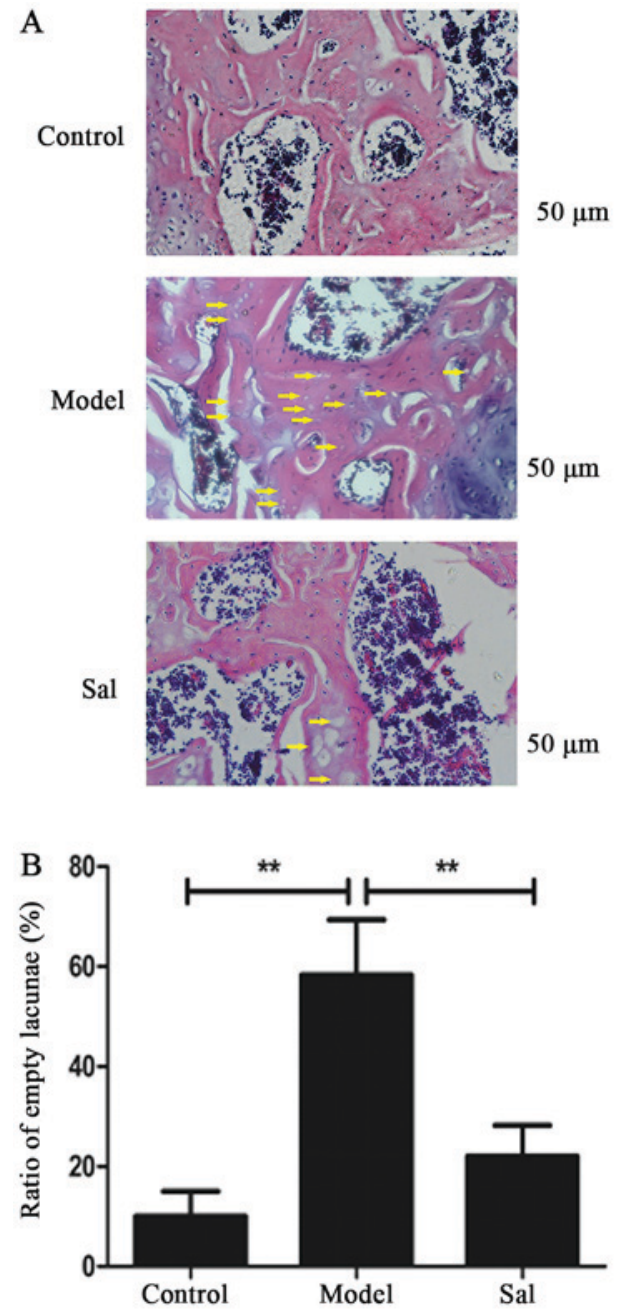

Figure 5. Histological analysis of rat femoral heads. The model group showed numerous empty lacunae surrounded by necrotic marrow cells (A). Fewer empty bone lacunae were observed in the Sal group compared to the model group. Empty lacunae are indicated by yellow arrows (B). The ratio of empty lacunae was significantly higher in the model group compared with the control and Sal groups. Data represent the average \pm standard deviation. Significant differences between two groups are indicated as ${ }^{* *} \mathrm{P}<0.01$. Sal, salidroside.

rate and decreasing cell apoptosis rate and $\mathrm{LDH}$ release rate. Furthermore, the change of TUNEL positive cells in TUNEL staining also proved our point. Sal decreased the expression of cleaved caspase-3, cleaved caspase-9, BAX, cytochrome C and increased Bcl-2 expression in Dex-induced osteoblasts apoptosis. Sal protected against Dex-induced osteoblasts apoptosis in part through the activation of the PI3K/Akt signaling pathway, which might result in the inhibition of caspase-3 and caspase-9 expression. In vivo, the reduction of empty lacunae in hematoxylin-eosin staining indicated Sal protected against SANFH.

Nowadays, Dex and other GCs have been long used for serious infection and immunological diseases and so on (3-6). However, lots of studies indicate Dex and other GCs can induce potential osteoblastic apoptosis which also leads to SANFH $(7,12)$. The mechanism of SANFH and the protective method against SANFH are still unclear. Our previous studies indicated that Dex could induce osteoblasts apoptosis and SANFH in rats or human specimens via activating
STAT1-mediated upregulation of caspase-3 (12,28). A recent study showed the activation of the PI3K/Akt signaling Pathway was important in anti-apoptosis of osteoblasts (25). The mechanism of anti-osteoblasts apoptosis seems to be not unique, and the PI3K/Akt signaling pathway plays an important role. Studies which focused on nerve cells showed the PI3K/Akt signaling pathway was a protective factor of Sal on anti-apoptosis $(19,29)$. However, the protective effect of Sal on anti-osteoblasts apoptosis has not been reported before. In our present study, we investigated the effects of Sal in osteoblasts in vitro and in a rat SANFH model in vivo.

Previous studies showed Dex and other GCs increased the expression of caspase protein family especially caspase- 3 which was related to osteoblasts apoptosis (30) and other cell apoptosis $(31,32)$. Caspase-3 is of great importance in osteoblasts apoptosis related to osteonecrosis (33), and the obvious elevation is one of the typical pathological characteristics of SANFH (28). The PI3K/Akt pathway is a very important component of cell survival, proliferation, and growth pathways in many different cell types including osteoblast $(19,29,30)$. Akt which serves as the substrate of PI3K plays a vital role in the antiapoptotic pathway $(34,35)$. Activated by PI3K, the Akt pathway caused the production of phosphatidylinositol trisphosphate and the phosphorylation of threonine-308 and serine-473 induces the activation of Akt (19). Activation of the PI3K/Akt pathway can prevent osteoblast against apoptosis, and the activated Akt can reduce the risk of cell injury during oxidative stress, free radical exposure and DNA damage $(19,36)$. Previous studies show Sal has an anti-apoptosis on cardiac H9c2 cells, PC12 cells and RSC96cells via activating the PI3K/Akt pathway $(19,37,38)$. In our present study, we found that the activation of the PI3K/Akt pathway played a crucial role in osteoblasts anti-apoptosis. We guessed that Sal downregulating cleaved caspase-3 might be related to the activation of the PI3K/Akt signaling pathway. However, the exact mechanism is still unclear and further studies shall be done to find out the truth.

Bcl-2 and BAX serve respectively as the antiapoptotic and proapoptotic regulators. And the imbalance between Bcl-2 and BAX, which stands for mitochondrial dysfunction, is responsible for Dex-induced osteoblasts apoptosis $(39,40)$. Mitochondria induce cellular apoptosis by the release of cytochrome C, and Sal can reverse it in Dex-induced osteoblasts apoptosis (41). Cytochrome $\mathrm{C}$ promote the generation of caspase-9, which subsequently activates caspase-3 expression (12). Our present study demonstrated that Sal showed up anti-apoptotic effect partly by inhibiting the mitochondrial pathway.

As described in our previous study (12), we adopted double dosage of LPS and MPS to generate a modified rat SANFH model. Previous study mentioned that the diffuse presence of empty lacunae in the bone trabeculae could stand for the severity of SANFH (27). Steffen et al found a significantly greater percentage of empty lacunae within the trabecular bone in the avascular necrosis of femoral necrosis (42). The empty lacunae represent the bone necrosis degree and reflect the effect of bone repair to some extent. As a result, we used hematoxylin-eosin staining to calculate the percentage of empty lacunae in rats to evaluate the effect of Sal on preventing 
SANFH. The reduction of empty lacunae in Sal treated group indicated Sal protected against SANFH.

In conclusion, our present study demonstrated that Sal alleviated Dex-induced osteoblasts apoptosis via activating the PI3K/Akt signaling pathway and downregulating caspase-3 expression in osteoblasts. Sal also protected against SANFH in SANFH rat models by decreasing the percentage of empty lacunae. The inhibition of the mitochondrial apoptosis pathway was involved. Further research is necessary to decipher the detailed mechanism of Sal in protecting against Dex-induced SANFH.

\section{Acknowledgements}

The authors thank the staff of the Laboratory of the Orthopaedic Research Institute and the Scientific Research Center of the Second Affiliated Hospital of Wenzhou Medical University. The present study was generously supported by grants from the Zhejiang Province Public Welfare Technology Application Research Project, China (2015C33209) and Wenzhou Public Welfare Science and Technology Project, Zhejiang Province, China (Y20150243).

\section{References}

1. Simon JP, Berger P and Bellemans J: Total hip arthroplasty in patients less than 40 years old with avascular necrosis of the femoral head. A 5 to 19-year follow-up study. Acta Orthop Belg 77: 53-60, 2011.

2. Motomura G, Yamamoto $\mathrm{T}$, Yamaguchi R, Ikemura $\mathrm{S}$, Nakashima Y, Mawatari T and Iwamoto Y: Morphological analysis of collapsed regions in osteonecrosis of the femoral head. J Bone Joint Surg Br 93: 184-187, 2011

3. Tanaka Y, Yoshikawa N, Hattori S, Sasaki S, Ando T, Ikeda M and Honda M; Japanese Study Group for Renal Disease in Children: Combination therapy with steroids and mizoribine in juvenile SLE: A randomized controlled trial. Pediatr Nephrol 25 877-882, 2010

4. Hodson EM and Alexander SI: Evaluation and management of steroid-sensitive nephrotic syndrome. Curr Opin Pediatr 20: $145-150,2008$

5. Tönshoff B, Höcker B and Weber LT: Steroid withdrawal in pediatric and adult renal transplant recipients. Pediatr Nephrol 20: 409-417, 2005.

6. Schäcke H, Döcke WD and Asadullah K: Mechanisms involved in the side effects of glucocorticoids. Pharmacol Ther 96: 23-43, 2002.

7. Nagasawa K, Tada Y, Koarada S, Tsukamoto H, Horiuchi T, Yoshizawa S, Murai K, Ueda A, Haruta Y and Ohta A: Prevention of steroid-induced osteonecrosis of femoral head in systemic lupus erythematosus by anti-coagulant. Lupus 15: 354-357, 2006.

8. Weinstein RS, Jilka RL, Parfitt AM and Manolagas SC: Inhibition of osteoblastogenesis and promotion of apoptosis of osteoblasts and osteocytes by glucocorticoids. Potential mechanisms of their deleterious effects on bone. J Clin Invest 102: 274-282, 1998

9. Kim HJ: New understanding of glucocorticoid action in bone cells. BMB Rep 43: 524-529, 2010.

10. Souttou B, Raulais D and Vigny M: Pleiotrophin induces angiogenesis: Involvement of the phosphoinositide-3 kinase but not the nitric oxide synthase pathways. J Cell Physiol 187: 59-64, 2001.

11. Himburg HA, Muramoto GG, Daher P, Meadows SK, Russell JL, Doan P, Chi JT, Salter AB, Lento WE, Reya T, et al: Pleiotrophin regulates the expansion and regeneration of hematopoietic stem cells. Nat Med 16: 475-482, 2010.

12. Feng Z, Zheng W, Tang Q, Cheng L, Li H, Ni W and Pan X: Fludarabine inhibits STAT1-mediated up-regulation of caspase-3 expression in dexamethasone-induced osteoblasts apoptosis and slows the progression of steroid-induced avascular necrosis of the femoral head in rats. Apoptosis 22: 1001-1012, 2017.

13. Chen X, Zhang Q, Cheng Q and Ding F: Protective effect of salidroside against $\mathrm{H}_{2} \mathrm{O}_{2}$-induced cell apoptosis in primary culture of rat hippocampal neurons. Mol Cell Biochem 332: 85-93, 2009.
14. Huang SC, Lee FT, Kuo TY, Yang JH and Chien CT: Attenuation of long-term Rhodiola rosea supplementation on exhaustive swimming-evoked oxidative stress in the rat. Chin J Physiol 52: 316-324, 2009.

15. Ma L, Cai D, Li H, Tong B, Song L and Wang Y: Anti-fatigue effects of salidroside in mice. J Med Coll PLA 23: 88-93, 2008.

16. Van Diermen D, Marston A, Bravo J, Reist M, Carrupt PA and Hostettmann K: Monoamine oxidase inhibition by Rhodiola rosea L. roots. J Ethnopharmacol 122: 397-401, 2009.

17. Zhang L, Yu H, Sun Y, Lin X, Chen B, Tan C, Cao G and Wang Z: Protective effects of salidroside on hydrogen peroxide-induced apoptosis in SH-SY5Y human neuroblastoma cells. Eur J Pharmacol 564: 18-25, 2007.

18. Chen X, Liu J, Gu X and Ding F: Salidroside attenuates glutamate-induced apoptotic cell death in primary cultured hippocampal neurons of rats. Brain Res 1238: 189-198, 2008.

19. Lu Z, Jiang G, Chen Y, Wang J, Muhammad I, Zhang L, Wang R, Liu F, Li R, Qian F and Li J: Salidroside attenuates colistin-induced neurotoxicity in RSC96 Schwann cells through PI3K/Akt pathway. Chem Biol Interact 271: 67-78, 2017.

20. Aziz SA, Davies M, Pick E, Zito C, Jilaveanu L, Camp RL, Rimm DL, Kluger Y and Kluger HM: Phosphatidylinositol-3-kinase as a therapeutic target in melanoma. Clin Cancer Res 15: 3029-3036, 2009.

21. Cantrell DA: Phosphoinositide 3-kinase signaling pathways. J Cell Sci 114: 1439-1445, 2001.

22. Gu YX, Du J, Si MS, Mo JJ, Qiao SC and Lai HC: The roles of PI3K/Akt signaling pathway in regulating MC3T3-E1 preosteoblast proliferation and differentiation on SLA and SLActive titanium surfaces. J Biomed Mater Res A 101: 748-754, 2013.

23. Guntur AR and Rosen CJ: The skeleton: A multi-functional complex organ: New insights into osteoblasts and their role in bone formation: The central role of PI3Kinase. J Endocrinol 211: 123-130, 2011.

24. Li L, Xia Y, Wang Z, Cao X, Da Z, Guo G, Qian J, Liu X, Fan Y, Sun L, et al: Suppression of the PI3K-Akt pathway is involved in the decreased adhesion and migration of bone marrow-derived mesenchymal stem cells from nonobese diabetic mice. Cell Biol Int 35: 961-966, 2011.

25. Wang J, Ma XY, Feng YF, Ma ZS, Ma TC, Zhang Y,Li X, Wang L and Lei W: Magnesium ions promote the biological behaviour of rat calvarial osteoblasts by activating the PI3K/Akt signaling pathway. Biol Trace Elem Res 179: 284-293, 2017.

26. Abdi A, Sadraie H, Dargahi L, Khalaj L and Ahmadiani A: Apoptosis inhibition can be threatening in $A \beta$-induced neuroinflammation, through promoting cell proliferation. Neurochem Res 36: 39-48, 2011.

27. Yamamoto T, Irisa T, Sugioka Y and Sueishi K: Effects of pulse methylprednisolone on bone and marrow tissues: Corticosteroid-induced osteonecrosis in rabbits. Arthritis Rheum 40: 2055-2064, 1997.

28. Xu X, Wen H, Hu Y, Yu H, Zhang Y, Chen C and Pan X: STAT1-caspase 3 pathway in the apoptotic process associated with steroid-induced necrosis of the femoral head. J Mol Histol 45: 473-485, 2014.

29. Zhang W, He H, Song H, Zhao J, Li T, Wu L, Zhang X and Chen J: Neuroprotective effects of salidroside in the MPTP mouse model of Parkinson's disease: Involvement of the PI3K/Akt/GSK3 $\beta$ pathway. Parkinsons Dis 2016: 9450137, 2016.

30. Dai WW, Wang LB, Jin GQ, Wu HJ, Zhang J, Wang CL, Wei YJ, Lee JH, Lay YE and Yao W: Beta-ecdysone protects mouse osteoblasts from glucocorticoid-induced apoptosis in vitro. Planta Med 83: 888-894, 2017.

31. Chua CC, Chua BH, Chen Z, Landy C and Hamdy RC: Dexamethasone induces caspase activation in murine osteoblastic MC3T3-E1 cells. Biochim Biophys Acta 1642: 79-85, 2003.

32. Ryu JS, Ko JH, Kim MK, Wee WR and Oh JY: Prednisolone induces apoptosis in corneal epithelial cells through the intrinsic pathway. Sci Rep 7: 4135, 2017.

33. Calder JD, Buttery L, Revell PA, Pearse M and Polak JM: Apoptosis-a significant cause of bone cell death in osteonecrosis of the femoral head. J Bone Joint Surg Br 86: 1209-1213, 2004.

34. Manning BD and Cantley LC: AKT/PKB signaling: Navigating downstream. Cell 129: 1261-1274, 2007.

35. Pap M and Cooper GM: Role of glycogen synthase kinase-3 in the phosphatidylinositol 3-kinase/Akt cell survival pathway. J Biol Chem 273: 19929-19932, 1998. 
36. Kang JQ, Chong ZZ and Maiese K: Critical role for Akt1 in the modulation of apoptotic phosphatidylserine exposure and microglial activation. Mol Pharmacol 64: 557-569, 2003.

37. Zhu Y, Shi YP, Wu D, Ji YJ, Wang X, Chen HL, Wu SS, Huang DJ and Jiang W: Salidroside protects against hydrogen peroxide-induced injury in cardiac $\mathrm{H} 9 \mathrm{c} 2$ cells via PI3K-Akt dependent pathway. DNA Cell Biol 30: 809-819, 2011.

38. Zhang L, Ding W, Sun H, Zhou Q, Huang J, Li X, Xie Y and Chen J: Salidroside protects $\mathrm{PC} 12$ cell from $\mathrm{MPP}^{+}$-induced apoptosis via activation of the PI3K/Akt pathway. Food Chem Toxicol 50: 2591-2597, 2012.

39. Li DW, Liu ZQ, Chen W, Yao M and Li GR: Association of glycogen synthase kinase-3 $\beta$ with Parkinson's disease (review). Mol Med Rep 9: 2043-2050, 2014.

40. Chao DT and Korsmeyer SJ: BCL-2 family: Regulators of cell death. Annu Rev Immunol 16: 395-419, 1998.
41. Hu WP, Yu HS, Sung PJ, Tsai FY, Shen YK, Chang LS and Wang JJ: DC-81-Indole conjugate agent induces mitochondria mediated apoptosis in human melanoma A375 cells. Chem Res Toxicol 20: 905-912, 2007.

42. Steffen RT, Athanasou NA, Gill HS and Murray DW: Avascular necrosis associated with fracture of the femoral neck after hip resurfacing: Histological assessment of femoral bone from retrieval specimens. J Bone Joint Surg Br 92: 787-793, 2010 .

(c) (i) $\odot$ This work is licensed under a Creative Commons EY NC ND Attribution-NonCommercial-NoDerivatives 4.0 International (CC BY-NC-ND 4.0) License. 